\title{
AVALIAÇÃO DA SOBRECARGA DO CUIDADOR FAMILIAR DE IDOSOS COM DEPENDÊNCIA FUNCIONAL
}

\section{EVALUATION OF FAMILY CAREGIVER OVERLOAD OF ELDERLY WITH FUNCTIONAL DEPENDENCE}

\section{Rodrigo Leite Rangel ${ }^{a *}$, Lucas Brito dos Santos ${ }^{b *}$, Elaine dos Santos Santana ${ }^{c * *}$, Maykon dos Santos Marinho ${ }^{\mathrm{d} * \star}$, Renato Novaes Chaves ${ }^{\mathrm{e}}$, Luciana Araújo dos Reis ${ }^{\mathrm{f} * *}$}

rodrigo.235@hotmail.coma , lucasbritorc@gmail.com ${ }^{b}$, elaine_137@hotmail.comc, mayckon_ufba@hotmail.comd,rnc_novaes@hotmail.com, lucianauesb@yahoo.com.br ${ }^{f}$ Faculdade de Tecnologia e Ciencias de Vitória da Conquista (FTC)*, Universidade Estadual do Sudoeste da Bahia (UESB)**

\section{RESUMO}

Introdução: O envelhecimento populacional vem acompanhado de uma série de alterações biopsicossociais que somadas as patologias que a acompanha, acaba favorecendo a situação de dependência, e com isso acaba necessitando de uma demanda de ações de cuidados da família ao idoso fragilizado. Objetivos: analisar o nível de sobrecarga do cuidador familiar de idosos com dependência funcional a partir do Questionário de Avaliação da Sobrecarga do Cuidador Informal, e investigar o perfil dos idosos e seus cuidadores familiares quanto às características sociodemográficas, econômicas e de saúde. Métodos: Foi um estudo epidemiológico, de base populacional, quantitativo, de corte transversal. Realizado na cidade de Vitória da Conquista - BA, com os idosos dependentes cadastrados no Programa de Atendimento Municipal Domiciliar ao Idoso com Limitação e seus cuidadores familiares, escolhidos por meio dos critérios de elegibilidade. Foram utilizados dois questionários, sendo um para os dados sociodemográfico e de avaliação das condições de saúde, bem como o Questionário de Avaliação da Sobrecarga do Cuidador Informal. Os dados foram tabulados com base no programa Statistical Package for Social Sciences. Resultados: prevalência de cuidadores do sexo feminino (87,3\%), filhas $(57,1 \%)$, casadas (41,3\%), com baixa escolaridade (50,8\%), no qual realizava a tarefa a mais de 10 anos (41,3\%). E quanto a sobrecarga $(85,7 \%)$ dos cuidadores familiares apresentam um quadro extremamente grave de sobrecarga. Conclusões: pode-se afirmar que a tarefa do cuidar traz um grande impacto no bem-estar físico, social, emocional e econômico do cuidador e de toda a família.

Palavras-chave: Idosos, envelhecimento, cuidadores, assistência domiciliar.

\section{ABSTRACT}

Introduction: Population aging is accompanied by a series of biopsychosocial changes that, together with the patologies that accompany it, end up favoring the situation of dependency, and with this end up necessitating a demand for family care actions for the frail elderly. Objectives: to analyze the level of overload of the family caregiver of the elderly with functional dependency from the Informal Caregiver's Overload Assessment Questionnaire, and to investigate the profile of the elderly and their family caregivers regarding socio-demographic, economic and health characteristics. Methods: It was an epidemiological, population-based, quantitative, cross-sectional study. Held in the city of Vitória da Conquista - BA, with the elderly dependents registered in the Program of Municipal Attention to the Elderly with Limitation and their family caregivers, chosen through the eligibility criteria. Two questionnaires were used, one for the sociodemographic data and for the evaluation of health conditions, as well as the Informal Caregiver Overload Assessment Questionnaire. Data were tabulated based on the Statistical Package for Social Sciences (SPSS) program. Results: prevalence of female caregivers (87.3\%), daughters (57.1\%), married (41.3\%), low educational level (50.8\%), performed the task more than 10 
years $(41.3 \%)$. As for the overload (85.7\%) of the family caregivers present an extremely severe picture of overload. Conclusions: it can be said that the task of caring has a great impact on the physical, social, emotional and economic well-being of the caregiver and the whole family.

Keywords: elderly, aging, caregivers, home care

\section{Introdução}

O envelhecimento da população é uma realidade e a projeção ${ }^{1}$, é de que até o ano de 2025, existirão aproximadamente 1,2 bilhões de pessoas idosas no mundo e, estima-se que neste mesmo período o Brasil passará a ser o sexto país do mundo em número de idosos².

O Brasil vive um processo de transição demográfica, em que o número de idosos cresce substancialmente. Este processo, por sua vez, é decorrente de diversos fatores, entre eles estão a redução de taxas de fecundidade e a diminuição da mortalidade e ampliação da expectativa de vida $^{2}$.

Dessa forma, constata-se que o tempo é um fator inexorável à vida humana e, uma de suas principais consequências é o envelhecimento, que traz consigo uma série de impactos para os indivíduos, como por exemplo, a diminuição da capacidade de realização das Atividades da Vida Diária (AVD) e o aumento das doenças crônicas não transmissíveis que repercutem de maneira significativa na vida destes ${ }^{3}$.

Nesse sentido, entende-se por envelhecimento como um processo natural, caracterizado por ganhos e perdas. No entanto, as modificações vêm acompanhadas de alterações de ordem biopsicossociais e da propensão destes ao aumento dos problemas de saúde. O indivíduo fica mais susceptível as doenças crônicas e isso favorece o risco de ter sua capacidade funcional prejudicada. Sendo assim, o idoso, quando acometido por um problema de saúde, necessita de cuidados, que geralmente ficam sob a responsabilidade da família ${ }^{4}$.

Em consequência do dano em sua capacidade funcional, o indivíduo fica impedido de executar tarefas cotidianas, necessitando de auxílio para o autocuidado e para conduzir sua vida normalmente. Devido essa realidade, os idosos passaram a exigir uma demanda de ações cuidativas, ficando a família responsável por assumir o papel de cuidador mesmo não tendo o preparo e a experiência para desempenhar essa atividade 4

Vale ressaltar que os principais cuidadores de idosos são representados pelo sexo feminino, geralmente são filhas ou cônjuges que prestam o cuidado. Ao assumir o papel de cuidadora, elas acabam abandonando toda sua vida, bem-estar social, saúde física e mental para prestar cuidados ao seu familiar ${ }^{5}$.

Neste contexto, o familiar por não possuir preparação adequada para assumir o papel de cuidador, acaba fazendo com que a sobrecarga caia toda sobre si, gerando assim um grande desgaste físico, crises de ansiedade, quadros depressivos que frequentemente afetam o seu bem-estar, sua saúde, autoestima e satisfação pela vida. Sendo assim, pode-se inferir que a qualidade de vida dos cuidadores é afetada devido à sobrecarga da tarefa do cuidar, uma vez que, eles ficam mais susceptíveis às doenças de ordem física e a síndromes psicológicas ${ }^{6}$.

Nesta perspectiva, esta pesquisa se ancora na possibilidade de inferir que o cuidado, quando mal executado, irá repercutir tanto na saúde idoso, quanto na qualidade de vida do cuidador que pode ter sobrecarga além do esperado. Assim, considerando que o envelhecimento promove alterações de toda ordem, apesar dos esforços para promover uma velhice mais ativa e saudável, muitos idosos experimentam algum tipo de incapacidade causada por patologias de agudas a crônicas. Nesse sentido, os resultados deste estudo podem trazer ganhos tanto para a saúde do cuidador familiar, como para o idoso, objeto do cuidado.

É neste cenário que surge este estudo, que se justifica pela necessidade de uma avaliação da sobrecarga do cuidador familiar de idosos com dependência funcional, assistidos pelo Programa 
de Atendimento Municipal Domiciliar ao Idoso com Limitação (PAMDIL). Os dados coletados servirão de base para a implementação de um protocolo de qualificação dos cuidadores e os aspectos relacionados à qualidade de vida dos idosos, dependentes nos níveis primário (Unidade de Saúde da Família-USF) e terciário (Hospital Geral de Vitória da Conquista/BA).

Este artigo é fruto do programa de Iniciação Científica e faz parte do projeto de produtividade em pesquisa de acesso ao nível PQ-2 para o período 2017 a 2019 intitulado "Qualificação dos cuidadores e aspectos relacionados a qualidade de vida dos idosos dependentes na atenção primária e terciária: proposição, implementação e avaliação de protocolo".

Tendo, portanto, como objetivos: analisar o nível de sobrecarga do cuidador familiar de idosos com dependência funcional a partir do Questionário de Avaliação da Sobrecarga do Cuidador Informal (QUASCI), e investigar o perfil dos idosos e seus cuidadores familiares quanto às características sociodemográficas, econômicas e de saúde.

\section{Material e Métodos}

Trata-se de uma pesquisa com delineamento epidemiológico de base populacional, com abordagem quantitativa, do tipo descritivo, com corte transversal, realizada no município de Vitória da Conquista, Bahia, com os idosos cadastrados no PAMDIL, que é responsável pelo atendimento de idosos com algum de tipo de limitação que são identificados pelo agente comunitário de saúde, tendo como referência uma Unidade de Saúde.

Vitoria da Conquista é uma cidade do interior baiano, pertencente à Região Sudoeste, que possui cerca de 350 mil habitantes. No município há um total de 42 unidades básicas de saúde, sendo 22 unidades na área urbana e 20 na zona rural. Destas 22 unidades básicas da área urbana, 14 unidades possuem Unidade de Saúde da Família totalizando 26 equipes.

Os participantes foram os cuidadores familiares dos idosos dependentes cadastrados no PAMDIL, tendo uma amostra de 63 indivíduos escolhidos por meio de critérios de inclusão, entre eles: ser membro da família do idoso; não receber nenhum tipo de remuneração para realizar a tarefa do cuidado; morar na mesma residência que o idoso, e ser o cuidador principal, sem restrição de gênero, estado civil e escolaridade.

Para a obtenção de dados, foi utilizado como instrumento da pesquisa, um questionário com variáveis sociodemográficas com data da aplicação, endereço, gênero, idade, escolaridade, estado civil, grau de parentesco, tempo de prestação de cuidados informais e renda familiar.

Ademais, tendo como foco a avaliação da sobrecarga do cuidador familiar, foi utilizado o Questionário de Avaliação da Sobrecarga do Cuidador Informal (QASCI), um instrumento composto por 32 itens, subdivididos em sete seções: Implicações na vida pessoal (11 itens); Sobrecarga financeira (2 itens); Sobrecarga emocional (4 itens); Satisfação com o papel e com o familiar (5 itens); Reações as exigências (5 itens); Percepção dos mecanismos de eficácia e de controle (3 itens) e Suporte familiar ( 2 itens).

Seus itens são avaliados por uma escala ordinal que varia de 1 a 5 categorias de respostas, que descrevem como cada questão afeta a pessoa, o escore total vai de 32 a 160 pontos e valores mais altos indicam maior sobrecarga vivenciada. A pontuação é dividida em ausência de sobrecarga para escores 0; escores de 1 a 25 sobrecarga leve; de 25 a 50 sobrecarga moderada; entre 50 a 75 sobrecarga grave e acima de 75 sobrecarga extremamente grave.

Para tabulação dos dados foi utilizado o Statistical Package for Social Sciences (SPSS), uma ferramenta informática, que permite a realização de cálculos estatísticos básicos e de complexidade, sendo então realizada a à análise estatística descritiva e inferencial.

Foi aprovado pelo Comitê de Ética em Pesquisa (CEP) da Universidade Estadual do Sudoeste da Bahia (UESB) no dia 20 de dezembro de $2016 \mathrm{sob}$ o parecer $n^{\circ} 1.875 .418$. 


\section{Resultados}

Na tabela 1, que traz o perfil sociodemográfico dos cuidadores familiares de idosos, evidenciouse uma maior frequência de adultos na faixa etária entre 18 a 59 anos $(69,9 \%)$. Sobre o gênero, 55
(87,3\%) são do sexo feminino, com 26 (41,3\%). Casados. Quanto a escolaridade, verificou-se que $32(50,8 \%)$ possuíam o ensino fundamental, evidenciando uma baixa escolaridade. Na profissão 29 (46\%) trabalham com serviços domésticos e quanto a religião 29 (46\%) eram evangélicos.

Tabela 1 - Distribuição percentual do perfil sociodemográfico dos cuidadores familiares dos idosos

\begin{tabular}{|c|c|c|c|}
\hline CATEGORIAS & VARIÁVEIS & F.A. & F.R. \\
\hline Idade & $\begin{array}{c}\text { Adultos (de } 18 \text { até } 59 \text { anos) } \\
\text { Idosos (60 anos ou mais) } \\
\text { Total }\end{array}$ & $\begin{array}{l}44 \\
19 \\
63\end{array}$ & $\begin{array}{l}69,9 \\
30,1 \\
\mathbf{1 0 0}\end{array}$ \\
\hline Gênero & $\begin{array}{c}\text { Masculino } \\
\text { Feminino } \\
\text { Total } \\
\end{array}$ & $\begin{array}{c}8 \\
55 \\
63\end{array}$ & $\begin{array}{l}12,7 \\
87,3 \\
\mathbf{1 0 0} \\
\end{array}$ \\
\hline Estado Civil & $\begin{array}{c}\text { Solteiro (a) } \\
\text { Casado/união estável (a) } \\
\text { Divorciado/separado (a) } \\
\text { Viúvo (a) } \\
\text { Total }\end{array}$ & $\begin{array}{c}18 \\
26 \\
11 \\
8 \\
63\end{array}$ & $\begin{array}{l}28,6 \\
41,3 \\
17,5 \\
12,7 \\
100\end{array}$ \\
\hline Escolaridade & $\begin{array}{c}\text { Ensino Fundamental } \\
\text { Ensino Médio (antigo } 2^{\circ} \text { grau) } \\
\text { Ensino Superior } \\
\text { Não estudou } \\
\text { Total } \\
\end{array}$ & $\begin{array}{c}32 \\
17 \\
10 \\
4 \\
63\end{array}$ & $\begin{array}{c}50,8 \\
27 \\
15,9 \\
6,3 \\
\mathbf{1 0 0}\end{array}$ \\
\hline Profissão & $\begin{array}{c}\text { Serviços domésticos } \\
\text { Funcionário público } \\
\text { Pedreiro/ajudante } \\
\text { Comércio } \\
\text { Aposentada } \\
\text { Estudante } \\
\text { Técnico em enfermagem } \\
\text { Autônomo } \\
\text { Total }\end{array}$ & $\begin{array}{c}29 \\
7 \\
2 \\
9 \\
4 \\
2 \\
2 \\
8 \\
63\end{array}$ & $\begin{array}{c}46,0 \\
11,1 \\
3,2 \\
14,3 \\
6,3 \\
3,2 \\
3,2 \\
12,7 \\
100\end{array}$ \\
\hline Religião & $\begin{array}{c}\text { Católico } \\
\text { Evangélico } \\
\text { Espiríta } \\
\text { Outra } \\
\text { Não tem } \\
\text { Total }\end{array}$ & $\begin{array}{c}26 \\
29 \\
3 \\
3 \\
2 \\
\mathbf{6 3}\end{array}$ & $\begin{array}{c}41,3 \\
46,0 \\
4,8 \\
4,8 \\
3,2 \\
\mathbf{1 0 0}\end{array}$ \\
\hline
\end{tabular}


A Tabela 2, apresenta o perfil familiar e as condições de saúde dos cuidadores de idosos. Com relação ao vínculo familiar, nota-se a predominância de quem presta os cuidados são os filhos 36 (57,1\%). Em relação a renda $41(65,1 \%)$ vive com a com um a dois salários mínimos, sendo a maior fonte desta renda a aposentadoria $39(61,9 \%)$. No que se refere a quantidade de indivíduos que moravam juntos, 35 (55,6\%) responderam que o arranjo familiar era composto de uma a três pessoas. E quanto aos problemas de saúde, as Doenças Crônicas Não Transmissíveis (DCNT) eram as que mais afetavam os cuidadores e, entre elas a Hipertensão 34 (54\%) e a Diabetes Mellitus 28 (44,4\%).

Tabela 2 - Distribuição percentual do perfil familiar e condições de saúde dos cuidadores dos idosos

\begin{tabular}{|c|c|c|c|}
\hline CATEGORIAS & VARIÁVEIS & F.A. & F.R. \\
\hline \multirow{6}{*}{ Vínculo Familiar } & Filho (a) & 36 & 57,1 \\
\hline & Irmão (a) & 7 & 11,1 \\
\hline & Cônjuge & 8 & 12,7 \\
\hline & Neto (a) & 7 & 11,1 \\
\hline & Sobrinha & 5 & 7,9 \\
\hline & Total & 63 & 100 \\
\hline \multirow{6}{*}{ Renda Familiar } & Sem renda & 2 & 3,2 \\
\hline & Até 1 salário mínimo* & 10 & 15,9 \\
\hline & De 1 a 2 salários mínimos & 41 & 65,1 \\
\hline & De 3 a 4 salários mínimos & 9 & 14,3 \\
\hline & Mais de 5 salários mínimos & 1 & 1,6 \\
\hline & Total & 63 & 100 \\
\hline \multirow{4}{*}{ Fonte de Renda } & Aposentadoria & 39 & 61,9 \\
\hline & Pensão & 4 & 6,3 \\
\hline & Outros & 20 & 31,7 \\
\hline & Total & 63 & 100 \\
\hline \multirow{5}{*}{$\begin{array}{c}\text { Número de pessoas que moram } \\
\text { juntas }\end{array}$} & 1 a 3 pessoas & 35 & 55,6 \\
\hline & 4 a 6 pessoas & 22 & 34,9 \\
\hline & 7 a 9 pessoas & 3 & 4,8 \\
\hline & Mais de 10 pessoas & 3 & 4,8 \\
\hline & Total & 63 & 100 \\
\hline \multirow{4}{*}{ Possui algum problema de saúde } & Hipertensão & 34 & 54,0 \\
\hline & Diabetes & 28 & 44,4 \\
\hline & Nenhum & 1 & 1,6 \\
\hline & Total & 63 & 100 \\
\hline
\end{tabular}

Fonte: Dados da Pesquisa, Vitória da Conquista - BA, 2018

F.A. Frequência Absoluta; F.R. Frequência Relativa.

Já a Tabela 3, apresenta os dados sobre o tempo de cuidado, o suporte social e a sobrecarga vivenciada pelo cuidador familiar. Em relação ao tempo de cuidado, foi verificado que $26(41,3 \%)$ executam esta atividade há mais de dez anos. Sobre o suporte social $55(87,3 \%)$ dos cuidadores familiares apontam que o nível deste suporte é médio. E quanto a sobrecarga avaliada pelo QASCI, $54(85,7 \%)$ dos cuidadores familiares apresentam um quadro extremamente grave de sobrecarga. 
Tabela 3 - Distribuição percentual do tempo de cuidado, suporte social e sobrecarga.

\begin{tabular}{|c|c|c|c|}
\hline CATEGORIAS & VARIÁVEIS & F.A. & F.R. \\
\hline \multirow{6}{*}{ Tempo de cuidado ao idoso } & Menos de um ano & 13 & 20,6 \\
\hline & 1 a 3 anos & 15 & 23,8 \\
\hline & 4 a 6 anos & 7 & 11,1 \\
\hline & 7 a 9 anos & 2 & 3,2 \\
\hline & Mais de dez anos & 26 & 41,3 \\
\hline & Total & 63 & 100 \\
\hline \multirow{4}{*}{ Suporte social } & Elevado suporte social & 1 & 1,6 \\
\hline & Médio suporte social & 55 & 87,3 \\
\hline & Baixo suporte social & 7 & 11,1 \\
\hline & Total & 63 & 100 \\
\hline \multirow{3}{*}{ Sobrecarga do QASCI } & Grave & 9 & 14,3 \\
\hline & Extremamente grave & 54 & 85,7 \\
\hline & Total & 63 & 100 \\
\hline
\end{tabular}

Fonte: Dados da Pesquisa, Vitória da Conquista - BA, 2018

F.A. Frequência Absoluta; F.R. Frequência Relativa.

\section{Discussão}

Ao analisar os resultados obtidos no presente estudo, pôde-se perceber que a tarefa do cuidar ainda está atrelada ao sexo feminino. Esses dados são semelhantes a um estudo ${ }^{7}$ realizado em Criciúma - SC, com a finalidade de avaliar a sobrecarga e a qualidade de vida de cuidadores da estratégia de saúde da família, no qual nota-se que 90,9\% dos cuidadores são do sexo feminino, o que evidencia uma maior tendência das mulheres na tarefa do cuidado.

Os dados deste estudo foram um pouco divergentes aos de uma pesquisa ${ }^{6}$ que mostrou a faixa etária prevalente dos cuidadores é superior a 60 anos. Ainda para os autores ${ }^{6}$, os cuidadores mais jovens podem sofrer maior sobrecarga devido a fatores como o isolamento, restrições sociais e de lazer, a quantidade de tempo direcionada ao cuidar e o pouco tempo para o autocuidado.

Com relação à escolaridade, pode-se perceber que grande parte dos entrevistados teve pouco acesso à educação e isso pode repercutir de maneira negativa na assistência e na qualidade de vida tanto do idoso quanto do cuidador. Outro estudo ${ }^{8}$ evidenciou que o cuidador de idoso deve ser uma pessoa capacitada, pois os idosos necessitam de cuidados complexos que demandam tempo e paciência. Para os autores, esses dados são um alerta, pois a falta de conhecimento pode levar os cuidadores e os idosos a sobrecargas físicas e emocionais.

Ademais, esta pesquisa demonstrou que os filhos que assumem o papel de cuidador familiar. Resultados e associações semelhantes podem ser vistos em outro resultado, no qual se pode observar que o papel de cuidador é assumido por pessoas que moram no mesmo domicílio e que geralmente são as filhas ou cônjuges.

Com relação a renda, observa-se que a maioria vive com um a dois salários mínimos. Sendo assim, pode-se inferir que os dados da pesquisa corroboram com outro estudo ${ }^{9}$, no qual traz dados de que devido à falta de recursos para contratar um cuidador, os próprios familiares se colocaram neste papel. Com isso, alguns destes abandonam seu trabalho para se dedicarem ao cuidado, eles passam a viver com a renda da pessoa cuidada o que é insuficiente para manter as necessidades do lar, do idoso e do cuidador. E isso contribui para 
que elas fiquem economicamente vulneráveis e consequentemente aumentando o seu nível de estresse, sobrecarga financeira e mental.

No que diz respeito às condições de saúde do cuidador, nota-se que a hipertensão e o diabetes mellitus são as doenças mais frequentes, o que pode modificar os padrões de vida, afetar o bemestar, a tarefa assistencial e a saúde do cuidador. Nesse sentido, um estudo ${ }^{10}$ sobre a saúde dos familiares cuidadores de idosos com doença crônica, mostra o impacto destas na vida do familiar cuidador e como ela contribui para o aumento da sobrecarga da tarefa do cuidar e da qualidade de vida. Sendo assim, a tarefa do cuidar torna-se ainda mais desgastante, estressante e difícil, pois a sobrecarga do cuidar acaba caindo sobre uma única pessoa e, assim deixa o indivíduo exposto ao desenvolvimento de incapacidades funcionais, cansaço físico e mental.

Ademais deixa os cuidadores mais vulneráveis as DCNT e as diversas patologias que podem favorecer situações de dependência, além de torna-los mais susceptíveis ao desenvolvimento de síndromes ansiosas ou depressivas ${ }^{11}$.

Já em relação a sobrecarga avaliada pelo QASCI, é percebido que a houve variância entre grave a extremamente grave, esses dados são compatíveis com uma pesquisa ${ }^{7}$ que estudou a sobrecarga no cuidado, estresse e impacto na qualidade de vida de cuidadores domiciliares, no qual pode-se notar que a sobrecarga está associada ao tempo e ao nível de dependência do idoso.

Quanto maior for a demanda do idoso, mais frequentemente o cuidador irá apresentar quadros de ansiedade e depressão e, com isso contribuindo para deterioração das suas condições físicas, sociais, emocionais e de saúde do cuidador ${ }^{7}$.

Em uma pesquisa ${ }^{12}$ realizada com 20 cuidadores em Foz do Iguaçu, Paraná, com o intuito de avaliar a qualidade de vida e a sobrecarga dos cuidadores, foi possível notar a presença de alteração em todas as subescalas do QASCI, esses dados corroboram com os desta pesquisa, no qual nota-se um índice de $81,1 \%$ de sobrecarga extremamente grave. Associado a isso, a dor e o despreparo, são fatores que acarretam as situações de sobrecarga para os cuidadores e relativamente gerando uma má qualidade de vida ${ }^{12}$.
Em outro estudo ${ }^{13}$, sobre as necessidades dos cuidadores familiares, observou-se que a medida que o tempo passa, os cuidadores vão ficando mais experiente ao desempenhar a tarefa do cuidar. Embora, ao se adaptar à situação, é necessário salientar sobre a importância do apoio familiar neste período, visto que o indivíduo passará por uma série de mudanças interpessoais, emocionais e sociais que afetam a sua saúde.

\section{Considerações Finais}

O estudo permitiu avaliar a sobrecarga do cuidador familiar de idosos com dependência funcional a partir do QASCI e comprovou que os cuidadores familiares participantes do estudo sofrem com a sobrecarga da tarefa do cuidar.

Ao traçar o perfil dos cuidadores nota-se a prevalência do sexo feminino, geralmente filhas, de baixa escolaridade e a maioria casada, com uma renda familiar entre um a dois salários mínimos.

Dito isso, não implica contradição afirmar que a tarefa do cuidar traz um grande impacto para a vida dos cuidadores familiares, principalmente aos que não tem qualificação, gerando maior, desgaste físico e consequente sobrecarga.

Sendo assim, devido à nova realidade do cotidiano do cuidador familiar é importante desenvolver medidas que possam aliviar a sobrecarga destes, visto que ela implica no bemestar físico, social, emocional e econômico do cuidador e de toda a família.

Ademais, estudos como este evidenciam a necessidade da implantação de políticas públicas, visando os cuidadores familiares e os idosos dependentes, tendo como medidas aplicáveis a melhoria da qualidade de vida, das condições de vida e, consequentemente diminuição da sobrecarga existente, das DCNT e dos gastos do município.

\section{Conflito de interesses}

Não há conflito de interesses para a condução da presente investigação. 


\section{Referências}

1. Organização Mundial de Saúde (OMS). Resumo: Relatório Mundial de Envelhecimento e Saúde. 2015. [25 de julho de 2018]. Disponível em: https://sbgg.org.br//wpcontent/uploads/2015/10/OMS-ENVELHECIMENTO2015-port.pdf

2. Chaves RN. Representações Sociais e Memória de idosos longevos sobre o processo de envelhecimento e a dependência funcional. Universidade Estadual do Sudoeste da Bahia, 2017. [19 de julho de 2018] Disponível em: http://www2.uesb.br/ppg/ppgmls/wp-content/ uploads/2017/06/Dissert.-Renato-Novaes-Chaves.pdf

3. Vanzella E, Nascimento JA, Santos SR. O envelhecimento, a transição epidemiológica da população brasileira e o impacto nas hospitalizações. Rev Elet Estácio Saúde. 2018; [citado 27 Jun 2018] 7(1). Disponível em: http://revistaadmmade.estacio.br/index.php/ saudesantacatarina/article/viewFile/3803/2033_

4. Santana, ES. Representações Sociais de idosos com dependência funcional sobre família: Memória das relações familiares. Universidade Estadual do Sudoeste da Bahia, 2017. [16 de julho de 2018] Disponível em: http://www2.uesb.br/ppg/ppgmls/wp-content/ uploads/2017/06/Dissert.-Elaine-dos-Santos-Santana. pdf

5. Reis LA, Santos KT, Gomes NP, Reis LA. Determinantes da sobrecarga e desconforto emocional em cuidadores de idosos. Revista Enfermagem Contemporânea. 2016; [citado 20 Jun 2018] 5(1):59-67. Disponível em: https:// www5.bahiana.edu.br/index.php/enfermagem/article/ view/888/648

6. Gratão ACM, Talmelli LFS, Figueiredo LC, Rosset I, Freitas CP, Rodrigues RAP. Sobrecarga e desconforto emocional em cuidadores de idosos. Texto Contexto Enferm, Florianópolis. 2012; [citado 17 Jun 2018] 21(2):304-312. Disponível em: http://www.scielo.br/pdf/ reeusp/v47n1/a17v47n1.pdf

7. Souza LR, Hanus JS, Libera LBD, Silva VM, Mangilli EM, Simões PW, et al. Sobrecarga no cuidado, estresse e impacto na qualidade de vida de cuidadores domiciliares assistidos na atenção básica. Cad. saúde colet. 2015; [citado 27 Jul 2018] 23(2)140-149. Disponível em: http://www.scielo.br/pdf/cadsc/v23n2/1414-462Xcadsc-23-2-140.pdf
8. Gutierrez LLP, Fernandes NRM, Mascarenhas M. Caracterização de cuidadores de idosos da região metropolitana de Porto Alegre (RS): perfil do cuidado. 2017; [citado 20 Jul 2018] 41(114):885-898. Disponível em: https://www.scielosp.org/article/ssm/content/ raw/?resource_ssm_path $=/$ media/assets $/$ sdeb/ v41n114/0103-1104-sdeb-41-114-0885.pdf

9. Almeida LPB, Menezes TMO, Freitas AVS, Pedreira LC. Características sociais e demográficas de idosos cuidadores e motivos para cuidar da pessoa idosa em domicílio. REME - Rev Min Enferm. 2018; [citado 25 Jul 2018] 22:e-1074.Disponível em: http://www.reme.org.br/ artigo/detalhes/1212

10. Vargas TB. O impacto das doenças crônicas na vida do familiar cuidador de idosos. Universidade Aberta dos SUS. Vitória, 2015. [26 de junho de 2018] Disponível: https://ares.unasus.gov.br/acervo/handle/ARES/8111

11. Faria AR. O cuidador e suas dificuldades no dia a dia: Revisão literária. Universidade Ferdal de Minas Gerais. Belo Horizonte, 2011. [25 de julho de 2018] Disponível: https://www.nescon.medicina.ufmg.br/biblioteca/ imagem/4867.pdf

12. Fernandes BCW, Ferreira KCP, Marodin MF, Val MON, Fréz AR. Influência das orientações terapêuticas na qualidade de vida e na sobrecarga dos cuidadores. Fisioter em Mov. 2013; [citado 27 Jul 2018] 26(1):151158. Disponível: https://periodicos.pucpr.br/index.php/ fisio/article/view/21540/20651

13. Fernandes CS, Angelo M. Family caregivers: what do they need? An integrative review. Rev Esc Enferm USP. 2016; [citado 15 Jul 2018] 50(4):672-678. Disponível: http://www.scielo.br/pdf/reeusp/v50n4/pt_0080-6234reeusp-50-04-0675.pdf

Como citar este artigo:

Rangel RL, Santos LB, Santana ES, Marinho MS, Chaves RN, Reis LA. Avaliação da Sobrecarga do Cuidador Familiar de Idosos com Dependência Funcional. Rev. Aten. Saúde. 2019;17(60):11-18. 